\title{
Degas' gähnende Büglerinnen zwischen Decorumsverstoß und Hysterie
}

\begin{abstract}
1901 schreibt Georg Simmel in seinem Aufsatz zur ästhetischen Bedeutung des Gesichts über barocke Pathosfiguren:

„Das Gefüge des Gesichts macht solche Zentrifugalität, d.h. Entgeistigung, von vornherein fast unmöglich. Wo sie einigermaßen stattfindet, beim Aufsperren des Mundes und dem Aufreißen der Augen, ist sie nicht nur ganz besonders unästhetisch, sondern gerade diese beiden Bewegungen sind, wie nun begreiflich ist, der Ausdruck des ,Entgeistertseins', der seelischen Lähmung, des momentanen Verlustes der geistigen Herrschaft über uns selbst. ${ }^{\text {"1 }}$
\end{abstract}

Erweitert um die psychoanalytische Folgerung repetiert der Philosoph und Soziologe hier nichts anderes als klassische Regeln des Decorums in der frühneuzeitlichen Bildkunst. ${ }^{2}$ Das Gesicht durch das übermäßige Öffnen von Mund oder Augen seiner Integrität zu berauben, entfernt den Künstler vom Ideal der angemessenen Darstellung. So wagt es Nicolas Poussin in seiner Blindenheilung aus dem Jahr 1650 zwar, die beinahe glotzenden - also ungläubig staunenden - Augen des alten Mannes im Bildzentrum zu zeigen, erlaubt sich jedoch, dessen offenstehenden Mund mit dem Arm Jesu zu verdecken. ${ }^{3}$ Gelten die Augen bereits in der Affektlehre der Renaissance als ,Fenster zur Seele“ und mithin als legitime Ausdrucksmittel, ${ }^{4}$ ist der Blick in die Mundhöhle ein klarer Decorumsverstoß; letzteres insbesondere, wenn dieser Einblick mit einem herzhaften Gähnen einhergeht.

So verwundert es kaum, dass sich vor dem 19. Jahrhundert nur wenige Darstellungen dieses eigentümlichen und rätselhaften Reflexes

1 Georg Simmel, „Die ästhetische Bedeutung des Gesichts“, in: Der Lotse. Hamburgische Wochenschrift für deutsche Kultur 35, 1901, S. 280-284, hier: S. 282.

2 Siehe hierzu bspw. Ursula Mildner, Das Decorum. Herkunft, Wesen und Wirkung des Sujetstils am Beispiel Nicolas Poussins, Sankt Augustin 1983.
3 Vgl. Lisa Hecht, „Verwunderung und Staunen als Primäraffekte in Nicolas Poussins ,Christus heilt die Blinden“ (1650)“, in: Marburger Jahrbuch, Marburg 2020, S. 151-165.

4 Vgl. Anna Degler, Parergon. Attribut, Material und Fragment in der Bildästhetik des Quattrocento, Paderborn 2015, S. 166. 


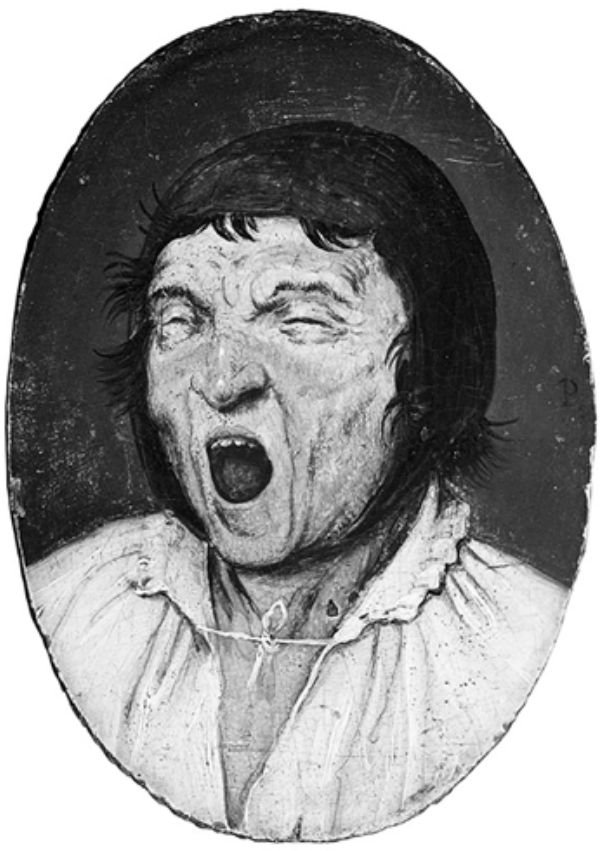

Abb. 1: Pieter Bruegel d. Ä. (nach?), Der Gähner, Öl auf Eichenholz, undatiert (1560er Jahre?), 12,6 × 9,2 cm, Königliche Museen der Schönen Künste Belgien, Brüssel

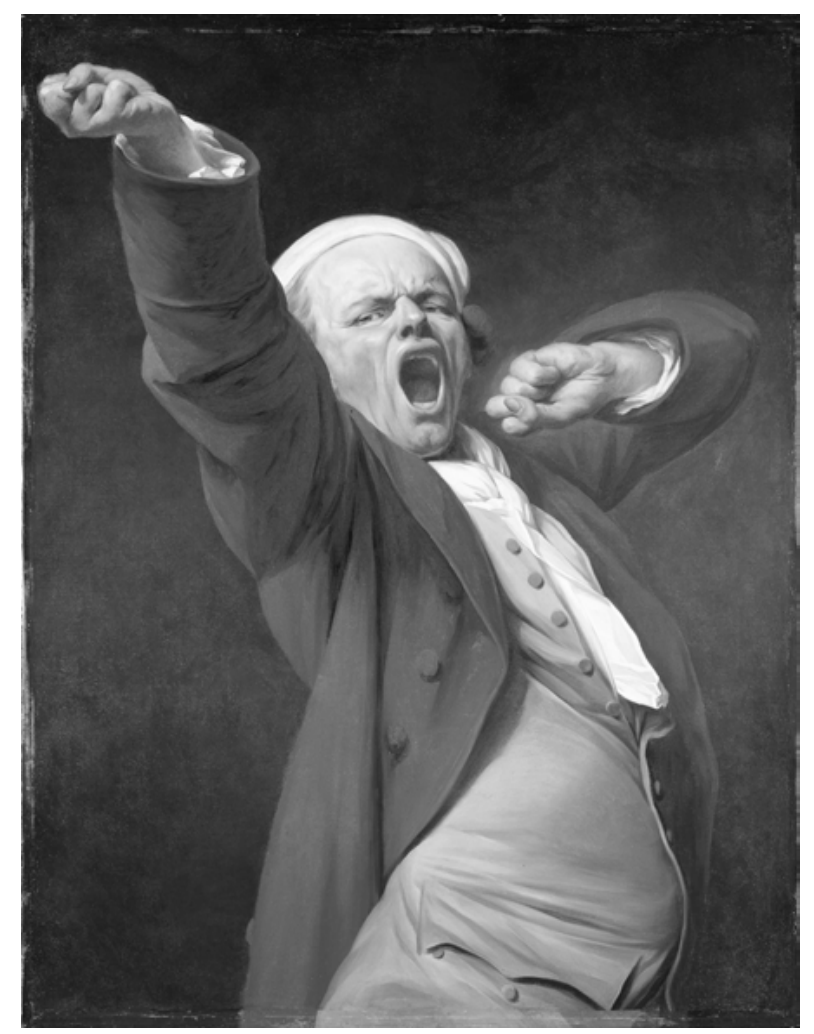

Abb. 2: Joseph Ducreux, Selbstportrait als Gähnender, Öl auf Leinwand, um 1783, 117,9 × 90,8 cm, J. Paul Getty Museum, Los Angeles 
auffinden lassen. Eine der frühesten im Medium des Tafelbildes dürfte der Pieter Bruegel d. Ä. zugeschriebene Gähner (17. Jhd.) in der Brüsseler Sammlung sein (Abb. 1). Möglicherweise spielt der Maler hier, wie so häufig in seinem (Euvre, auf ein bereits damals geläufiges Sprichwort an, welches besagt, wenn einer gähnt, gähnt der Andere. ${ }^{5}$

Hier liegt indes zunächst kein Decorumsverstoß vor, da Bruegel das verzerrte Gesicht des Gähnenden eindeutig einem Mann aus dem Bauernstand zuordnet und ihn so im geläufigen Kosmos der Genremalerei einbettet. Die Darstellung lässt sich in den Kontext der sogenannten Tronies einordnen; ${ }^{6}$ eine vor allem niederländische Gattung, die es zudem erlaubt, die menschliche Physiognomie im Gemälde auf ihre Extreme hin zu erproben. Angesichts der zwar kleinen, aber preziös geformten Tafel provoziert Bruegel die Frage der Bildwürdigkeit.

Ein weiterer Künstler, der die Betrachtenden umso offensiver mit dieser Frage konfrontiert, ist Joseph Ducreux, der sich mehr als 200 Jahre später in Lebensgröße als Gähnender selbst portraitiert (Abb. 2). Obwohl erfolgreicher premier peintre de la reine, wird Ducreux nie ordentliches Mitglied der Académie royale de peinture et de sculpture; dies wohl aufgrund seiner wenig anpassungsfähigen Persönlichkeit. ${ }^{7}$ Diese manifestiert sich nicht zuletzt in einem regelrechten Akt subversiver Extravaganz. ${ }^{8}$ Nachdem ihm die Präsentation seiner Gemälde im jährlichen Salon du Louvre verwehrt wurde, reagiert Ducreux mit einem ungewöhnlichen Selbstportrait im Salon de la Correspondance: In einem roten Hausmantel und mit einer Nachtkappe - als wäre er gerade erst aufgestanden - präsentiert der Künstler sich mitten im Akt des Gähnens, exakt so, wie es zuletzt der Verhaltensforscher Desmond Morris in seiner Anthologie der menschlichen Körperhaltungen beschrieben hat: „Yawning involves a combination of opening the jaws to the maximum, while inhaling and then exhaling through the gaping mouth, and is often accompanied by stretching movements of the neck, chest and arms. " ${ }^{9}$ In der bewussten Überschreitung der Darstellungsnorm des Menschen im gemalten Bild sind sowohl Bruegel als auch Ducreux Vertreter eines frühneuzeitlichen Bildwitzes. Es ist also davon auszugehen, dass beide Werke eine ähnliche Wirkung hatten, die sich

5 Vgl. Walter S. Gibson, Pieter Bruegel and the Art of Laughter, London 2006, S. 56.

6 Vgl. Lyckle de Vries, „Tronies and Other Single Figures Netherlandish Paintings“, in: Leids Kunsthistorisch Jaarboek 8, 1989/90, S. 185-202, hier: S. 191.

7 Vgl. Constance Naubert-Riser, „Joseph Ducreux“, in: The Great Parade. Portrait of the Artist as Clown, Ausst.-Kat. National Gallery of Canada, Ottawa 2004, S. 90 .
8 Zu Ducreux' Bâilleur und dessen immanenter Kritik an der Institution des Salons haben sich zuletzt Cornelia Logemann und Ulrich Pfisterer geäußert. Siehe Cornelia Logemann/Ulrich Pfisterer, „Kunst zum Gähnen! Joseph Ducreux' Selbstporträts“, in: Maria Effinger et al. (Hg.), Von analogen und digitalen Zugängen zur Kunst. Festschrift für Hubertus Kohle zum 60. Geburtstag, Heidelberg 2019, S. 131-139.

9 Desmond Morris, Postures. Body Language in Art, London 2019, S. 294. 
bei heutigen Betrachter*innen ebenfalls entfaltet. Auf Irritation folgt Belustigung angesichts des insbesondere im Fall von Ducreux mit dem Pathos des repräsentativen Bildnisses vorgetragenen Anti-Affekts. Der Franzose verbirgt daher hinter einem vordergründigen Bildwitz eine tiefgehende Subversion der akademisch geprägten Ästhetik. Als gähnender Laokoon verkehrt er die Pathosformel des geöffneten Mundes in ihr Gegenteil: aus dem exemplum doloris (,Schmerzensvorbild') wird ein exemplum satietatis (,Müdigkeitsvorbild').

Ducreux kann hier bereits an einen proto-soziologischen wie auch proto-neurologischen Diskurs seines Jahrhunderts anschließen. Gähnen gilt im Sinne der höfischen Etikette als Zeichen moralischer Entgleisung und zudem als Affront gegen die Mächtigen. ${ }^{10}$ Im Kontext der Aufklärung entsteht weiterhin die erste und bis heute immer noch geläufige Hypothese, Gähnen fördere die Sauerstoffzufuhr vom Blutstrom zum Gehirn. ${ }^{11}$ Nach wie vor ist nicht eindeutig geklärt, welche Funktion Gähnen im menschlichen Körper bzw. im sozialen Kontext einnimmt. In der neurologischen Forschung lassen sich hierzu drei Kernhypothesen ausmachen: Erstens die soziale Funktion des Gähnens; vor allem die ansteckende Wirkung ließe auf Gähnen als wichtigen Baustein im Empathiegefüge schließen; zweitens der seit dem 18. Jahrhundert nur leicht modifizierte Ansatz, Gähnen diene der Gehirnkühlung; drittens die Idee, Gähnen aktiviere das Aufmerksamkeitszentrum im Gehirn. ${ }^{12}$

Thema dieses Aufsatzes ist das Gähnen als soziales Zeichen im ausgehenden 19. Jahrhundert. Als eine der wenigen körperlichen Ausdrucksformen, die linguistische und nationale Grenzen überschreitet, rangiert das Gähnen laut Peter Toohey und Eran Dorfman zwischen den widersprüchlichen Zuständen der Langeweile und Erschöpfung. ${ }^{13}$ Dorfman führt hierzu weiter aus: „[Yawning means, l.h.] a certain strain or difficulty to be fully, there'; a secret wish to escape the present situation in favour of a different activity, be it of a higher or lower energetic nature, that is, either towards more adventure or rest. " ${ }^{14}$ Eben diese Dichotomie von Langeweile und Ermüdung ist auch für Edgar Degas' Gemälde Repasseuses (1884-86) von großer Bedeutung (Abb. 3).

10 Vgl. Wolf Lepenies, Melancholie und Gesellschaft, Frankfurt am Main ${ }^{3} 1998$ [1969], S. 56 und S. $62 f$.

11 Vgl. Olivier Walusinski, „Historical Perspectives“, in: ders. (Hg.), The Mystery of Yawning in Physiology and Disease. Front Neurol Neurosci, Basel 2010, S. 1-21, hier: S. 6-10.

12 Vgl. Ronald Baenninger, „On Yawning and Its Functions“, in: Psychonomic Bulletin \& Review 4, 1997, S. 198-207, hier: S. 205; Francis Schiller, „Yawning?“, in: Journal of the History of the Neurosciences 4 (11), 2002, S. 392-401, hier: S. 393.

$13 \mathrm{Vgl}$. Eran Dorfman, „Everyday Life between Boredom and Fatigue“, in: Michael E. Gardiner/Julian Jason Haladyn (Hg.), Boredom Studies Reader. Frameworks and Perspectives, London/New York 2017, S. 180-192; Peter Toohey, Boredom. A Lively History, New Haven/ London 2012, S. 11.

14 Dorfman 2017 (wie Anm. 13), S. 180. 


\section{Harte Arbeit, Erotik und Langeweile: Degas' Repasseuses}

Zwei Frauen stehen hinter einem schräg in den Bildraum hineinragenden Tisch, auf dem sich rechts ein weißes Wäschestück befindet, das soeben mit viel Kraftaufwand von der rothaarigen Büglerin in rosa Jacke und blauer Schürze geglättet wird. Ihre benachbarte Kollegin hat indes keine Textilien vor sich, sondern eine offenbar beinahe geleerte Weinflasche, die sie am Hals packt, während sie den anderen Arm zu ihrem Kopf führt. Es handelt sich bei dieser Geste um das Strecken der Gliedmaßen, welches mit dem ebenfalls im Bild sichtbaren Gähnen der Frau einhergeht. Der flimmernde Duktus, in dem Degas die Farbe aufträgt, thematisiert in diesem Gemälde ebenso die schwüle Luft im Raum und somit das erschwerte Atmen im sauerstoffarmen Dunst.

Degas setzt sich hier nicht zum ersten Mal mit dem Sujet der Büglerinnen auseinander, obwohl das Gemälde aus dem Musée d'Orsay wohl die größte Popularität in diesem Werkkomplex genießt. Seine erste Repasseuse fertigt er, inspiriert von Darstellungen Honoré Daumiers, um 1869 an (Abb. 4)..$^{15}$ Alexander Eiling zufolge handelt es sich aber noch eher um ein Rollenportrait denn um die Darstellung einer spontan eingefangenen Begebenheit des Pariser Milieus. Degas' Lieblingsmodell, Emma Dobigny, scheint im Münchener Bild noch an der Contenance der Alten Meister geschult, die der Maler zeitlebens intensiv studiert und rezipiert. ${ }^{16}$ Frontal ist sie in einigermaßen aufrechter Pose den Betrachtenden zugewandt und doch verrät ihr Gesichtsausdruck eine Müdigkeit, die sie zugleich äußerst sinnlich inszeniert. Diese Verbindung von Erschöpfung und Erotik ist in vielen Frauenbildern Degas' als männliche Projektion auf die hart arbeitenden Frauen zu verstehen. ${ }^{17}$

Die zwischen 12 und 18 Stunden pro Tag tätigen Büglerinnen des späten 19. Jahrhunderts stehen laut Gabriela Baumann sozial etwas über den noch schlimmeren Zuständen ausgesetzten Wäscherinnen, sind jedoch ebenfalls schlecht bezahlt und so häufig gezwungen, der Prostitution als Nebenerwerb nachzugehen..$^{18}$ Nicht nur deshalb erwecken sie Vorstellungen von sexueller Verfügbarkeit. Wegen der ständigen Hitze leicht bekleidet und in gebückter Haltung sind ihre Körper nur zu häufig durch die geöffneten Fenster im Souterrain den männlichen Blicken ausgesetzt. Dies schlägt sich nicht zuletzt in einer Varieté-Revue 


\section{Lisa Hecht}

Degas' gähnende Büglerinnen zwischen Decorumsverstoß und Hysterie

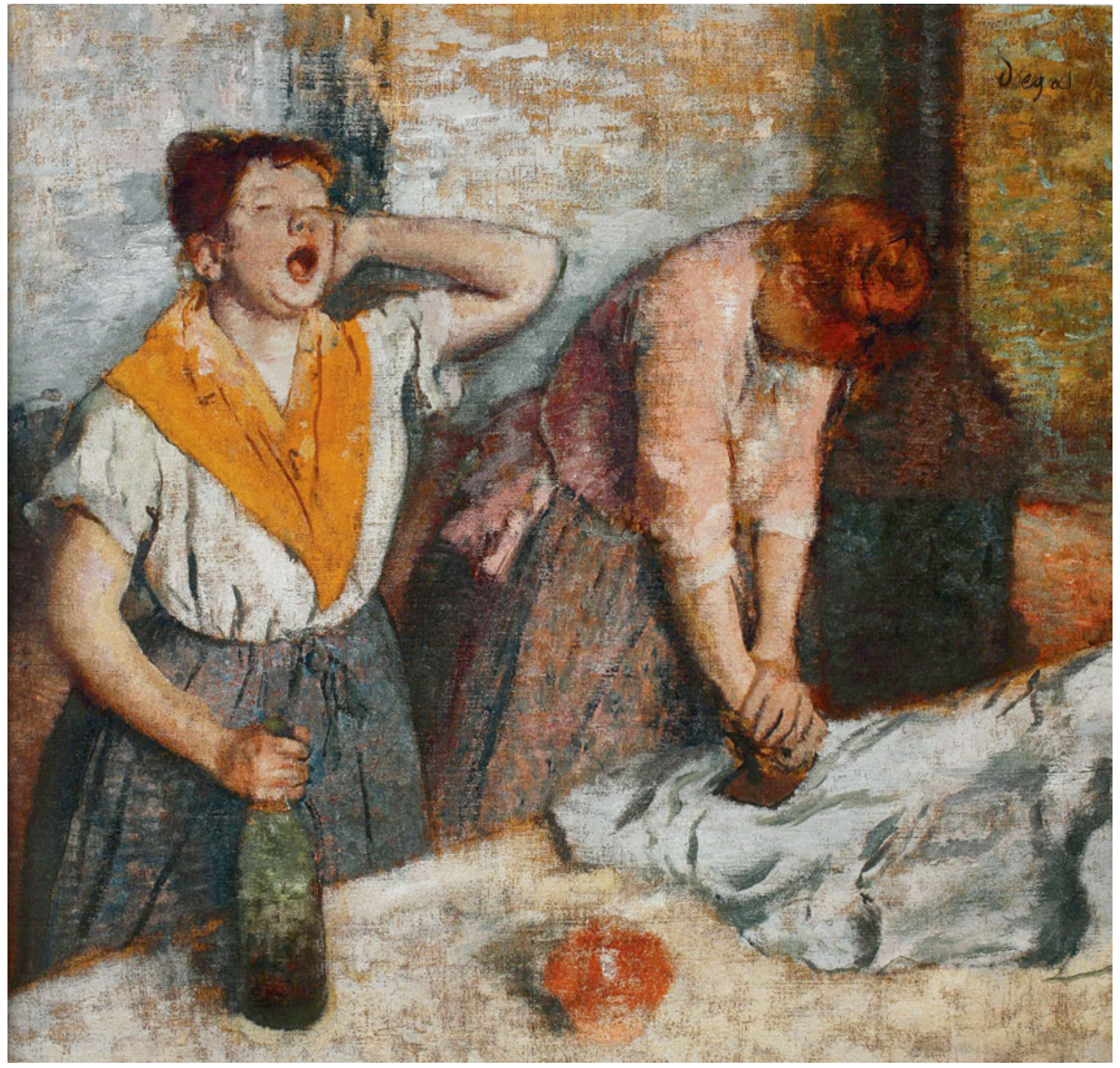

Abb. 3: Edgar Degas, Die Büglerinnen (Repasseuses), Öl auf Leinwand, 1884-86, 76 × 81,5 cm, Musée d'Orsay, Paris 

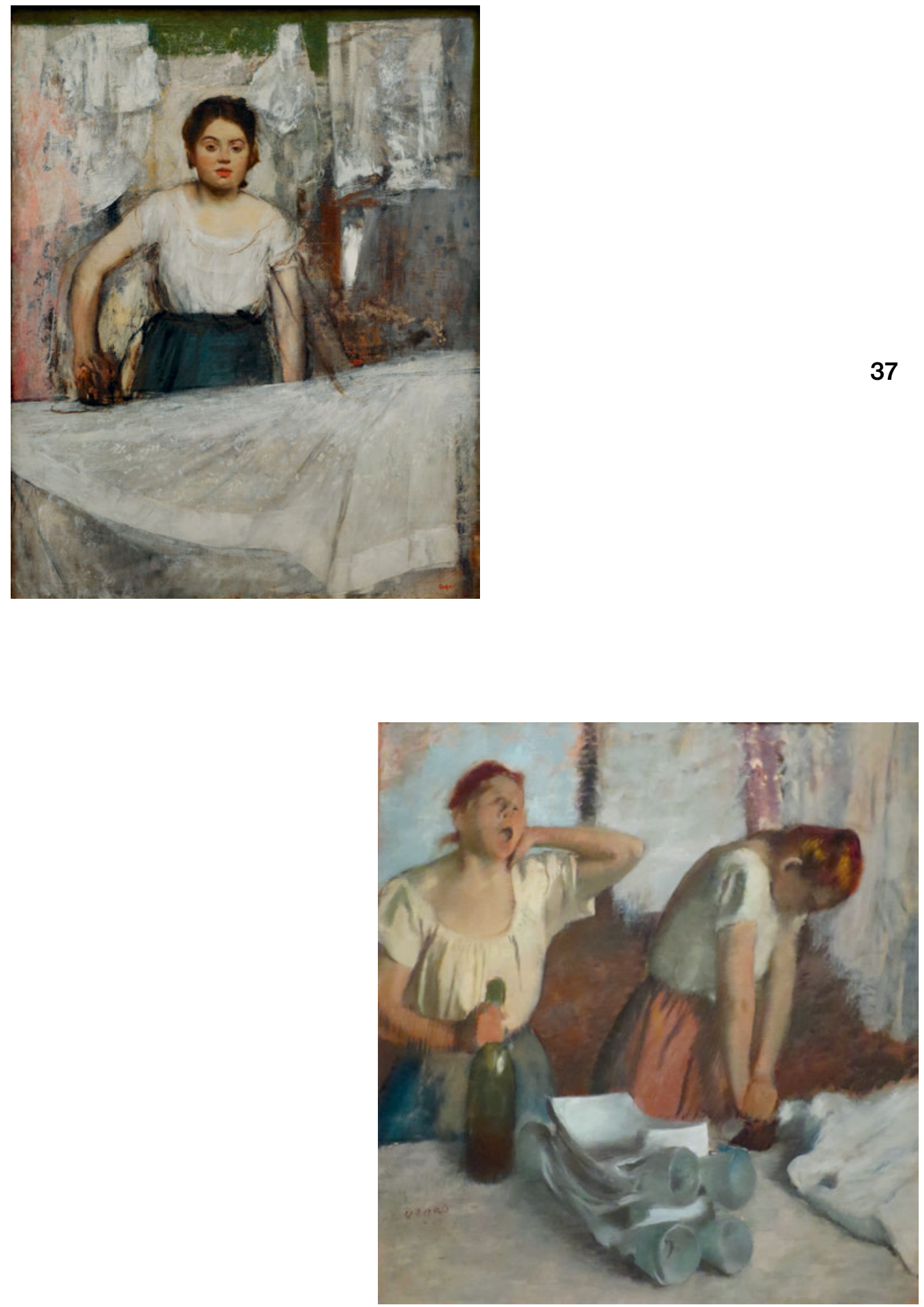

Abb. 4: Edgar Degas, Büglerin, Öl auf Leinwand, 1869, $92,5 \times 73,5 \mathrm{~cm}$, Neue Pinakothek München
Abb. 5: Edgar Degas, Büglerinnen, Öl auf Leinwand, 1882-86, $81,9 \times 75,5 \mathrm{~cm}$ Norton Simon Museum, Pasadena, Kalifornien 
aus dem Jahr 1860 nieder, die Schlüssellochblicke auf hingebungsvolle Wäscherinnen mit entblößten Brüsten präsentiert. ${ }^{19}$

Diese heute vernachlässigte Lesart der Büglerinnen in den Bildern Degas' lässt sich auf Basis des literarischen Diskurses des 19. Jahrhunderts begründen. Noch in den 1890er Jahren schreibt der französische Autor und Verleger Octave Uzanne über Büglerinnen, die sich $\mathrm{zu}$ den niedrigsten Formen der Prostitution gezwungen sahen. ${ }^{20}$ Die aus bürgerlich männlicher Sicht schamlose Büglerin wird nicht zuletzt in Émile Zolas Erzählung „L'Assommoir“ (1877) zum Topos:

\begin{abstract}
„Il faisait là une température à crever. On avait laissé ouverte la porte de la rue, mais pas un souffle de vent ne venait. [...] Depuis un instant, sous cette lourdeur de fournaise, un gros silence [...]. Tout d'un coup, elle s'écria : Ah ! non, mademoiselle Clémence, remettez votre camisole. Vous savez, je n'aime pas les indécences. Pendant que vous y êtes, montrez toute votre boutique. Il y a déjà trois hommes arrêtés en face. La grande Clémence la traita de vieille bête, entre ses dents. Elle suffoquait, elle pouvait bien se mettre à l'aise [...]. Et elle levait les bras, sa gorge puissante de belle fille crevait sa chemise, ses épaules faisaient craquer les courtes manches." ${ }^{21}$
\end{abstract}

Insbesondere in einer zweiten Version des Gemäldes wird der mögliche Bezug zu Zolas Schilderung bei Degas deutlich (Abb. 5). Die Bildanlage und Komposition wird größtenteils beibehalten, doch handelt es sich bei der gähnenden Büglerin um einen leicht veränderten Frauentypus. Schlanker in der Figur und vor allem leichter bekleidet als in der Pariser Version erscheint sie wie eine Schwester der schamlosen Clémence aus Zolas Roman. Zwar ist auch hier nicht der von Madame Putois gescholtene Einblick in Sicht, jedoch handelt es sich meiner Meinung nach um eine von Degas' häufigen subtilen erotischen Anspielungen. ${ }^{22}$ Nicht die entblößte Brust verweist auf die Schamlosigkeit der Arbeiterin, sondern ihr zum Gähnen geöffneter Mund, den sie zudem nicht einmal mit ihrer Hand verdeckt.

19 Vgl. Hofmann 2007 (wie Anm. 15), S. 162.

20 Vgl. Eunice Lipton, „The Laundress in Late NineteenthCentury French Culture. Imagery, Ideology and Edgar Degas“, in: Art History 3 (3), 1980, S. 295-313, hier: S. 295.

21 Émile Zola, L'Assomoir, Paris 1951, S. 175-176, dt.: Émile Zola, Der Totschläger, übers. v. Gerhard Krüger, München 1975, S. 208-210: „Es herrschte eine Temperatur zum Umkommen. Man hatte die Tür zur Straße offengelassen, aber kein Windhauch wehte herein. [...] Seit einer Weile herrschte in dieser Backofenschwüle tiefe Stille [...] Auf einmal rief [Frau Putois, I.h.]: ,Ach nein, Mademoi- selle Clémence, ziehen Sie Ihre Unterjacke wieder an. Wissen Sie, ich habe Unanständigkeiten nicht gern. Wenn Sie so dastehen zeigen Sie ja Ihren ganzen Kramladen. Da drüben sind schon drei Männer stehengeblieben.' Die lange Clémence schalt sie, zwischen den Zähnen brummelnd, eine alte Ziege. Sie erstickte ja, sie könne es sich doch bequem machen [...]. Und sie hob die Arme, ihr mächtiger Busen, der Busen eines schönen Mädchens, zerplatzte schier ihr Hemd, ihre Schultern brachten die kurzen Ärmel zum Krachen.“

22 Vgl. Wendy Lesser, His Other Half. Men Looking at Women Through Art, Cambridge/London 1991, S. 78 . 
Die Soziologin Franziska Lamott hat bereits auf die erotisch-sexuelle Implikation des Oralen um 1900 hingewiesen: „Der Mund gehört zweifellos zu den intimen Körperöffnungen und ist als einzige von ihnen unverhüllt. [...] Die Bedeutsamkeit der Oralität für die Libidoentwicklung gehört zum Wissensbestand der Jahrhundertwende. ${ }^{23}$ Der Blick in die Mundhöhle ist somit nicht nur ein künstlerischer Decorumsverstoß, sondern weist darüber hinaus in die Richtung expliziter Erotik. Der weibliche Mund öffnet sich, um den männlichen Kuss zu empfangen; der gähnende Mund der Büglerin assoziiert somit eben jene sexuelle Verfügbarkeit, die Zola oder die Pariser Revue den hart arbeitenden Frauen unterstellt.

Der Theater- und Medienwissenschaftler Lorenz Aggermann bezeichnet den offenen Mund im Bild als eine Leerstelle, vergleichbar mit dem onomatopoetischen Laut im Text:

„Diese ,Leerstellen' geben, egal mit welchen Zeichen oder Lauten man sie füllt, ihren affektiven Gehalt nicht adäquat wieder, sie widersetzen sich einer sprachlichen, sprich syntaktischen wie semantischen Logik und reißen Löcher in den Text, da sich ihr Inhalt nicht adäquat erfassen, abbilden oder zur Sprache bringen läßt.“24

In Bezug auf die Schreie im Theater heißt es weiter: „Pathos muß folglich unweigerlich mit ,Leerstellen“ Hand in Hand gehen, da es die Sphäre des Menschen übersteigt, sich diesem nur als Chaos offenbart." 25 Der zum Schrei geöffnete Mund ist also vor allem Ausdruck eines mit den Mitteln der Sprache nicht einholbaren Gefühls: Pathos. Der zum Gähnen geöffnete Mund hat im stummen Bild vieles mit dem Blick in die schreiende Mundhöhle gemein, doch negiert er gleichsam das Pathos. Während geöffneter Mund und erhobene Arme in der vormodernen Kunst zumeist für einen narrativen Höhepunkt eingesetzt sind, in dem Laute und Rufe das Geschehen untermalen, werden dieselben Gesten bei Degas zur piktoralen Manifestation des Vulgären oder des Alltäglichen. ${ }^{26} \mathrm{Ob}$ das Gähnen der Büglerin nun ein Symptom der Müdigkeit oder der Langeweile ist - in jedem Fall scheint es im

23 Franziska Lamott, Die vermessene Frau. Hysterien um 1900, München 2001, S. $143 f$.

24 Lorenz Aggermann, Der offene Mund. Über ein zentrales Phänomen des Pathischen, Berlin 2013, S. 8.

25 Ebd., S. 9.

26 Siehe hierzu auch Degas' Chanteuse de Café (um 1878), Pastell auf Leinwand, 53,2 × $41 \mathrm{~cm}$, Harvard Art

Museum/Fogg Museum. 
Gemälde eine Subversion des tradierten Anspruchs der Kunstproduktion zu sein, Interesse auszulösen, indem Interessantes dargestellt wird. Die Leerstelle verweist somit ganz konkret auf eine inhaltliche Leere, eine Erfahrung ohne Inhalt. ${ }^{27}$ Das Interesse am an sich belanglosen Bildinhalt ergibt sich außerhalb des Bildes: die erotische Aufladung der Szene entsteht durch die Genese eines voyeuristischen Blickwinkels. Die Arbeiterin in Degas' Bild fühlt sich unbeobachtet. Auch ihre Kollegin ist ganz in die Arbeit vertieft. Degas' Vorliebe für eine voyeuristische Rezeptionshaltung verbindet sich also mit dem dezidiert unpathetischen, dennoch narrativen Inhalt zu einer Hinterfragung des Bildwürdigen, wie dies mehrfach in den letzten Jahrzehnten des 19. Jahrhunderts geschieht.

Auch die Zeitgenossen diskutieren Degas' Umgang mit den Regeln des Decorums in diesem Werk kontrovers. Noch 1929 schreibt der Kunstkritiker Paul Jamot in La Peinture au Musée du Louvre: „C'est une ronde commère qui attrape d'une main une bouteille et, de l'autre, cale sa joue distendue par un bâillement à décrocher, comme on dit, la mâchoire." 28 Jamot spielt zudem auf ein weiteres Motiv an: die vor allem im Pariser Bild prominent inszenierte Weinflasche. Nicht zuletzt in Zolas Roman wird der verbreitete Alkoholismus unter Wäscherinnen und Büglerinnen ebenfalls zum Topos. Während die gefalteten Hemden in der Fassung des Norton Simon-Museums zumindest noch den Anschein einer Arbeitspause erwecken, fehlt dieses Versatzstück gänzlich im Pariser Bild. Hier trinkt die Büglerin also nicht mehr aus Entkräftung nach getaner Arbeit, sondern scheinbar grundlos.

Somit scheint das Gähnen im Pariser Bild nicht aus Erschöpfung zu resultieren. Ist es stattdessen der profane Ausdruck von Langeweile? Büglerinnen dürften zwar kaum gelangweilt gewesen sein; zugleich handelt es sich bei ihrer harten Arbeit allerdings um eine äußerst monotone Tätigkeit, geprägt von den immer gleichen Abläufen. Erschöpfung und Langeweile könnten somit gemeinsam auftreten, wobei deren sichtbare Äußerung gleichermaßen das Gähnen ist. Was aus heutiger Sicht wie eine realistische und sozialkritische Schilderung des harten Arbeitslebens anmutet, wird erst unter Einbezug historischer Geschlechterdiskurse verständlich. Vor diesem Hintergrund löst sich die nur scheinbare Paradoxie des Zugleich von Erschöpfung und Langeweile auf.

27 Siehe weiterführend Elizabeth Goodstein, Experience Without Qualities. Boredom and Modernity, Stanford 2005.
28 Paul Jamot, La Peinture au musée du Louvre. École française. XIXe siècle (Troisième Partie), Paris 1929, S. 71, dt. [l.h.]: „Das ist eine dicke Klatschbase, die mit einer Hand eine Flasche greift und sich mit der anderen die Wange hält, die von einem Gähnen aufgebläht ist, das ihr beinahe den Kiefer aushakt.“ 


\section{Hysterie: Die Pathologisierung der gelangweilten Frau}

Der Topos der gelangweilten Frau hat eine lange kulturelle Geschichte. Noch in Reinhard Kuhns Standardwerk The Demon of Noontide. Ennui in Western Literature (1976) wird klar zwischen dem geistigen Ennui des männlichen Genies und der pathologisierten Langeweile der Frau unterschieden. ${ }^{29}$ Während Langeweile beim Mann also als Anzeichen von intellektueller Ermüdung angesichts einer für das Genie unerträglich prosaisch gewordenen Gesellschaft interpretiert wird, ist die gelangweilte Frau stets auf ihren vermeintlich unbeschäftigten Körper zurückgeworfen. Zwar bietet die Salonkultur des 17. und 18. Jahrhunderts einige Beispiele selbstreflexiver Texte von sozial hochstehenden Damen, die über eben diesen Zustand referieren, ${ }^{30}$ doch bleibt es lange bei der von Kuhn beschriebenen Sicht einer patriarchal geprägten Gender-Diskrepanz im Langeweile-Diskurs. Auch das Gähnen der Büglerinnen ist kein Ausdruck dekadenter Gleichgültigkeit, sondern von körperlicher Erschöpfung und „Entgeistigung“, wie Simmel sie beschreibt.

Der Begriff der „Entgeistigung“ wird umso relevanter im Hinblick auf die diskursive Verortung in der Entstehungszeit von Degas' gähnenden Büglerinnen. Im Hysterie-Verständnis der zweiten Hälfte des 19. Jahrhunderts subsumieren sich die unterschiedlichsten Krankheitsbilder. So wird insbesondere die weibliche Langeweile oder Melancholie mit der Erkrankung eines vermeintlich überaktiven Uterus in Zusammenhang gebracht. ${ }^{31}$ Die gelangweilte Hausfrau wird ebenso schnell Opfer der Hysterie wie die ungebildete Arbeiterin aufgrund ihrer körperlichen Erschöpfung und geistigen Unreflektiertheit.

Bereits 1815 assoziiert der Chirurg Augustin Landré-Beauvais Gähnen mit diversen pathologischen Zuständen: „Les attaques de goutte, d'hystérie, d'hypochondrie, et même celles d'épilepsie, s'annoncent assez souvent par un bâillement continuel." ${ }^{32}$ Auch im ersten enzyklopädischen Medizinlexikon, das in 60 Bänden zwischen 1812 und 1822 in Paris erscheint, wird Gähnen als unwillkürliche Handlung den konvulsivischen Erkrankungen wie Epilepsie und Hysterie zugesprochen. ${ }^{33}$ Am berühmtesten dürfte jedoch Charles Darwins Beschreibung des Gähnens in seiner Abhandlung The Expression of the Emotions in

29 Vgl. Reinhard Kuhn, The Demon of Noontide. Ennui in Western Literature, Princeton/New Jersey 1976, S. 7.

30 Man denke hierbei an die Briefe der Marquise de Maintenon oder Liselottes von der Pfalz am Hof Ludwigs XIV., an die wiederkehrenden Aussagen der aufklärerischen Salonnière Madame du Deffand über ihren unerträglichen Ennui oder an die Tagebucheinträge und Korrespondenzen Lady Mary Wortley Montagus sowie der englischen Autorin Frances Burney. Vgl. hierzu: Lepenies 1998 (wie Anm. 10), S. 194; Patricia Meyer Spacks,
Boredom. The Literary History of a State of Mind, Chicago 1995, S. 57-73, S. 84-93 und S. 102-105.

$31 \mathrm{Vgl}$. Alison Pease, Modernism, Feminism and the Culture of Boredom, Cambridge 2012, S. 6 und S. 24.

32 Augustin Jacob Landré-Beauvais, Séméiotique, ou traité des signes des maladies, Paris ${ }^{3} 1818$ [1809], S. 85, dt. [I.h.]: „Anfälle von Gicht, Hysterie, Hypochondrien und sogar von Epilepsie werden häufig durch ein kontinuierliches Gähnen angekündigt“.

33 Vgl. Walusinski 2010 (wie Anm. 11), S. 11. 
Man and Animals (1872) sein: „The abnormal action of the voluntary muscles in epilepsy, chorea, and hysteria is known to be influenced by the expectation of an attack, and by the sight of other patients similarly affected. So it is with the involuntary acts of yawning and laughing." ${ }^{34}$

Somit ist die Repräsentation einer gähnenden Frau im 19. Jahrhundert eng mit dem Assoziationsfeld der Hysterie verbunden und bettet sich in die Ikonografie der Hysterie ein, welche sich in den 1870er und 80er Jahren ausbildet. ${ }^{35}$ Allen voran die Publikationen aus der psychiatrischen Anstalt La Salpêtrière in Paris, unter der Leitung von Jean-Martin Charcot, haben einen bedeutenden Anteil an dieser Entwicklung.

Schon für Degas' Darstellungen von badenden Frauen hat Carol Armstrong 2003 festgestellt, dass sie möglicherweise Bezugspunkte zu Paul Richers Illustration von Verrenkungen des weiblichen Körpers in Charcots Études cliniques sur la Grande Hystérie (1885) aufweisen könnten. ${ }^{36}$ Und auch das Gähnen selbst wird nur wenig später zu einem bildwürdigen Untersuchungsgegenstand, als sich Charcot der Fotografie zuwendet.

Am 23. Oktober 1888 präsentiert der leitende Arzt der Salpêtrière seinem Auditorium einen außergewöhnlichen Fall. Seine 17-jährige Patientin gähnt achtmal in der Minute, also 480mal in der Stunde, und unterbricht dies lediglich, wenn sie schläft. Dieses Verhalten wurde mit dem hysterisch motivierten Drang der Patientin verbunden, ihre Nervenzentren mit Sauerstoff zu versorgen. ${ }^{37}$ Heute gehen Neurologen davon aus, dass die junge Frau an einem gutartigen Tumor litt, der auf Sehnerv und Hypothalamus drückte. ${ }^{38}$ Abgebildet wird dieser Fall wahrscheinlich im selben Jahr in der Nouvelle Iconographie de la Salpêtrière (Abb. 6), in einer dreiteiligen beinahe chronofotografischen Serie. Stets im selben Bildausschnitt ist eine junge Frau in einem hochgeschlossenen Kleid zu sehen, die lockigen Haare weitestgehend aus dem Gesicht genommen. Alles an dieser Reihe soll den objektiv wissenschaftlichen Geist evozieren, der vermeintlich allen fotografischen Aufnahmen aus der Salpêtrière eigen ist. Doch irritiert vor allem der Fakt, dass das Modell die Gliedmaßen nicht streckt und die Augen

34 Charles Darwin, The Expression of the Emotions in Man and Animals, London ${ }^{2} 1890$ [1872], S. 360.

35 Die Kultur- und Literaturwissenschaftlerin Elizabeth Bronfen hat den Ursprung und die Vorbilder dieser Ikonografie der Hysterie bereits in Darstellungen „von dämonischer Besessenheit und von Teufelsaustreibung“ verortet: Elizabeth Bronfen, Das verknotete Subjekt. Hysterie in der Moderne, Berlin 1998, S. 259; vgl. Georges Didi-Huberman, Erfindung der Hysterie. Die photographische Klinik von Jean-Martin Charcot, München 1997, S. 55 und Nico Kirchberger, Schau(spiel) des Okkulten. Die Bedeutung von Mesmerismus und Hypnotismus für die bildende Kunst im 19. Jahrhundert, Berlin 2016, S. 155f.

36 Vgl. Carol Armstrong, Odd Man Out. Readings of the Work and Reputation of Edgar Degas, Los Angeles 2003, S. 186.

37 Vgl. Schiller 2002 (wie Anm. 12), S. 39; Walusinski 2010 (wie Anm. 11), S. 15.

38 Vgl. Walusinski 2010 (wie Anm. 11), S. 15. 
nicht schließt - äußerst ungewöhnlich für das offenbar herzhafte Gähnen mit weit geöffnetem Mund, das hier präsentiert wird.

Wie Georges Didi-Huberman für die Bilder aus der Salpêtrière festgestellt hat, handelt es sich auch hier um die Reinszenierung und damit Ästhetisierung eines angeblich hysterischen Zustandes. „Wissenschaft also," wie Didi-Huberman anmerkt, „die sich aus einem Paradox herstellt: Sie produziert ihre theoretische Legitimation durch Figuren aus der bildenden Kunst, das heißt, durch Figuren aus dem Bereich der Fiktion. “39 Aufgrund ihres höchst artifiziellen, inszenierten Charakters wirken insbesondere die Bilder, welche Paul Régnard von Charcots favorisierter Patientin Augustine anfertigt, bis in die Avantgarde des 20. Jahrhunderts nach. André Breton, der am Ende seines Romans Nadja konstatiert: "La beauté sera CONVULSIVE ou ne sera pas“, ${ }^{40}$ erklärt die Fotografien aus der Salpêtrière 1928 zum ästhetischen Ausgangspunkt des Surrealismus. ${ }^{41}$ Transzendenz im Sinne der körperlich-geistigen Überschreitung wird der surrealistischen Kunst und Literatur zum Diktum und die Patientinnen Charcots zu ihren Idealfiguren.

\section{Ästhetik des Konvulsiven}

Die modernistische Entgrenzung wird in der zunehmenden Bildwürdigkeit des Konvulsiven im 19. Jahrhundert eingeleitet. Zunächst unter dem Deckmantel der medizinischen Objektivität vorgetragen, beeinflussen die Darstellungen aus der Salpêtrière alsbald auch die arrivierten Bildkünste der Salons. Bereits Georges Guillain zählt in seiner Biografie Charcots mehrere zeitgenössische Künstler auf, welche die sogenannten „leçons de mardi“ (Dienstagsvorlesungen) besuchten ${ }^{42}$ und Deborah Silverman vermutet in Auguste Rodin einen der illustren Gäste in Charcots Privathaus, wo der Arzt zu wöchentlichen Salons lud. ${ }^{43}$ Einflüsse der Hysterieforschung auf die Kunst wurden allerdings bisher weitestgehend auf Werke seit den frühen 1890er Jahren nachgewiesen; man denke nur an Jean-Léon Gérômes La Vérité (1896) oder Giovanni Segantinis Le cattive madri (1896/97) ${ }^{44}$

39 Georges Didi-Huberman, „Ästhetik und Experiment bei Charcot. Die Kunst, Tatsachen ins Werk zu setzen“, in: Wunderblock. Eine Geschichte der modernen Seele, Ausst.-Kat. Historisches Museum Wien 1989, S. 281-296, hier: S. 282. In Les Démoniaques dans l'art argumentiert Charcot selbst allerdings gegen eine solche Lesart. Vielmehr geht er davon aus, dass Momente der Erleuchtung und der religiösen Verzückung in Kunstwerken stets Abbilder hysterischer Zustände waren. Vgl. Jean Martin Charcot/Paul Richer, Les Démoniaques dans l'art, Paris 1887, insbes.: S. V-XII.
40 André Breton, Nadja, Paris ${ }^{18} 1949$ [1928], S. 215, dt.: André Breton, Nadja, übers. v. Bernd Schwibs, Frankfurt am Main 2002, S. 139: „Die Schönheit wird KONVULSIV sein oder nicht sein".

41 Vgl. Louis Aragon/André Breton, „Le Cinquantenaire de I'hystérie“, in: La Révolution Surréaliste 4, 1928, S. 20-22.

42 Vgl. Georges Guillain, J.-M. Charcot 1825-1893. Sa vie son ouvre, Paris 1955, S. 36.

43 Vgl. Deborah L. Silverman, Art Nouveau in Fin-de-Siècle France. Politics, Psychology, and Style, Berkeley u.a. 1989, S. 243-269.

44 Vgl. Kirchberger 2016 (wie Anm. 35), S. 172-175. 


\section{Lisa Hecht}

Degas' gähnende Büglerinnen zwischen Decorumsverstoß und Hysterie

44

Nouvelle Iconographie de LA SALPÊtriére

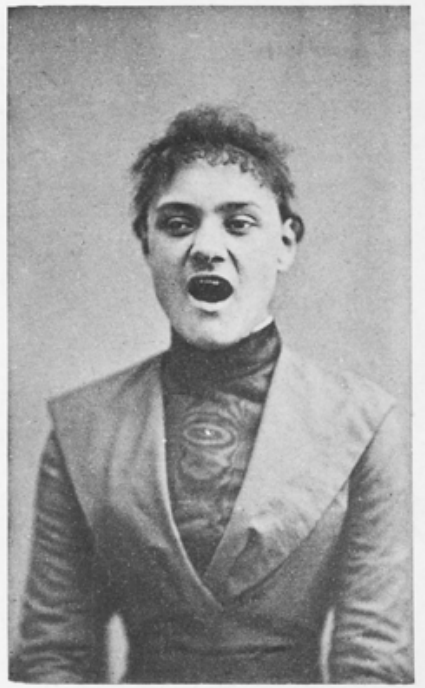

PHototvPE NÉATtr A. LONDE

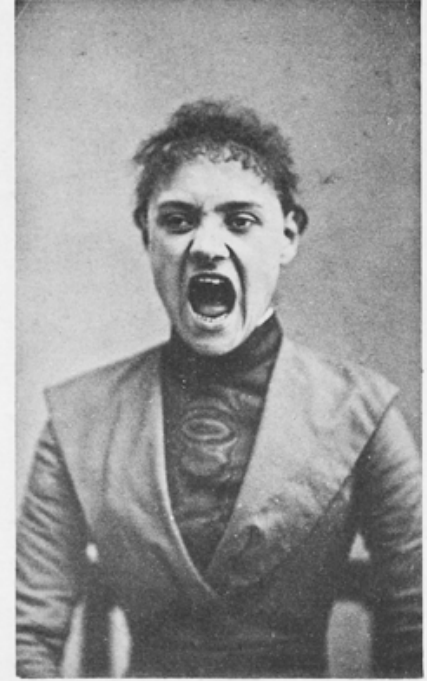

BAILLEMENTS HYSTÉRIQUES
T. III. PL. XVIII

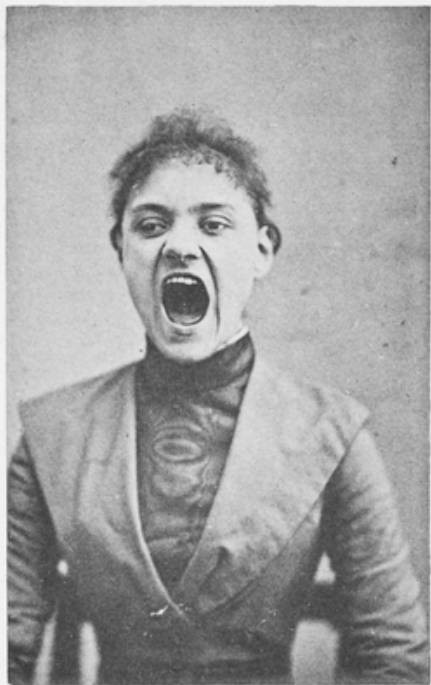

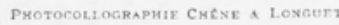

LECrosNiFr a BAnk, ÉDTTXUR

Abb. 6: Albert Londe, Bâillement Hystériques, in:

Nouvelle Iconographie de la Salpetrière, Paris 1888 

atroce font dela bouche l'organe des cris d'chirants, 1930 
Degas' Büglerinnen stehen am Anfang dieser Entwicklung. Der Maler kann zwar noch nicht auf den bildlichen Diskurs zum hysterischen Gähnen (Albert Londes Fotografie entsteht ca. fünf Jahre nach Degas' Repasseuses), wohl aber auf die bestehenden zeitgenössischen Hysterie-Theorien, zurückgreifen. Außerdem hat sich gezeigt, dass bereits vor der Entstehung von Degas' Werk mehrere soziologische, psychologische und auch belletristische Werke immer wieder auf eine Verbindung von Hysterie, genuin weiblicher Langeweile sowie Passivität, und den Affekt des Gähnens abheben. Texte wie jene Zolas und Darwins bereiten die pathologischen Schlussfolgerungen der Hysterieforschung vor. Degas' Repasseuses reihen sich also in eine diskursive Entwicklung ein, die in den hochgradig inszenierten Fotografien der gähnenden Patientin Charcots kulminieren.

Degas scheint insbesondere an der körperlichen Entgrenzung interessiert. Dies schließt zunächst an die traditionelle Wahrnehmung des malerischen Decorumsverstoßes an. Für den Impressionisten hingegen wird die gähnende Arbeiterin zum modernistischen Sujet einer Kunstauffassung, die sich um Momenthaftigkeit bemüht und zugleich Großstadtphänomene der Entfremdung, Anonymität und Distanz kritisch hinterfragt. Sie ist aber auch eine genuin weibliche Figuration des 19. Jahrhunderts: Ihr aufgerissener Mund ist noch das alles verschlingende Gähnen des romantischen und industrialisierten Zeitalters. Erst 50 Jahre später öffnet sich dieser Mund erneut zum entgrenzenden Schrei der Avantgarde des 20. Jahrhunderts (Abb. 7). 